\title{
Monitoring Risk Among Injection Drug Users in New Orleans-Findings from New Orleans National HIV Behavioral Surveillance 2005-2012
}

\author{
Kiva Fisher*1, Narquis Barak ${ }^{1}$, Meagan C. Brown ${ }^{3}$ and William T. Robinson ${ }^{2,3}$ \\ ${ }^{1}$ NO/AIDS Task Force, New Orleans, LA, USA; '2Louisiana Office of Public Health STI/HIV Program, Office of Public Health, Louisiana, \\ LA, USA; ${ }^{2}$ Louisiana State University Health Sciences Center, Louisiana, LA, USA
}

\section{Objective}

The objective of this study is to present findings from the National HIV Behavioral Surveillance (NHBS) in New Orleans. Local data focused on the injection drug using (IDU) population was analyzed using representative samples from three years, 2005, 2009, and 2012, to garner an understanding of high-risk behavior associated with disease transmission.

\section{Methods}

The CDC funded National HIV Behavioral Surveillance System is being implemented in 20 cities throughout the United States, including the New Orleans Metropolitan Statistical Area (MSA). Each site conducts research in one year cycles to measure behaviors of three populations at high risk for HIV transmission; Men who have sex with men (MSM), injection drug users (IDU), heterosexuals at high risk for HIV (HET). Respondent Driven Sampling (RDS) is used to collect a representative sample of IDU within the population. Individuals who report having injected drugs in the past 12 months and are eligible, complete an in-depth interview and are offered an HIV test. Data were analyzed using SPSS. Respondents were interviewed about their sexual and drug use behaviors for all cycles ( $\mathrm{n}=372$ in $2005, \mathrm{n}=$ 619 in 2009, $\mathrm{n}=495$ in 2012).

\section{Results}

Overall, findings indicate a generalized epidemic among IDU in New Orleans, showing a higher than $1 \%$ positivity. The positivity rate has increased incrementally, from $4 \%$ in self-reported positivity to $6 \%$ and $9 \%$, with results verified by a rapid HIV test with Western blot confirmatory. There has also been an increase in the percentage of IDU identifying as 'bisexual,' ( $6 \%$ in $2005,10 \%$ in $2009,12 \%$ in 2012). A rise in the average number of MSM partners appears. At the same time, there has been a decline in the number of heterosexual female partners among men. In contrast, the average number of heterosexual male partners among females has increased dramatically. Regarding high risk drug use behavior, there appears to be an ascending trend in needle sharing behavior, and the most recent cycle indicates that sharing injection equipment has also increased in recent years. In conjunction with this finding, there also is a rise in self-reported Hepatitis cases. No striking observable trend appears among demographic factors, although the percentage of white injectors has expanded since 2005 .

\section{Conclusions}

These results indicate an increase an increase in high-risk sexual and drug use behavior among Injection Drug Users (IDU) in New Orleans since 2005. These data show that particular focus should be directed towards MSM behavior and sharing of injection equipment (including syringes). These behaviors put IDU at risk for HIV. Furthermore, risky injection practices appear to be increasing, putting IDU at risk for Hepatitis $\mathrm{C}$ and other adverse health outcomes, which is further indicated in the escalating cases of self-reported Hepatitis cases. These results show that there is a trend in high risk behavior among IDU that needs to be addressed. Focus should be redirected when targeting the IDU population to curb high risk sexual and drug use behavior to reduce disease transmission. Through analyzing three representative samples of IDU in the New Orleans MSA over time, we are better able to understand trends in demographic and risk behavior specific to our community.

\section{Keywords}

HIV; High-Risk Behavior; Injection Drug Use; Surveillance

\section{Acknowledgments}

The authors would like to acknowledge support through the Centers for Disease Control and Prevention (CDC) for National HIV Behavioral Surveillance cooperative agreement number $5 \mathrm{U} 1 \mathrm{~B} / \mathrm{PS} 003252$. Its contents are solely the responsibility of the authors and do not necessarily represent the official views of the CDC.

\section{*Kiva Fisher}

E-mail: kivaf@noaidstf.org 